\title{
Sperm plasma membrane characteristics and boar semen fertility
}

\author{
R. A. P. Harrison \\ Department of Signalling, The Babraham Institute, Babraham, Cambridge CB2 4AT, UK
}

\begin{abstract}
Much effort is being made to establish relationships between the molecular events that take place in spermatozoa under fertilizing conditions and actual sperm function during fertilization. During capacitation, the process that 'primes' spermatozoa for interaction with the egg, components of the sperm's environment, notably bicarbonate, provoke various specific changes in the architecture and functioning of the sperm plasma membrane in a large number of cells. The individual changes have been found to proceed on different time scales, and may therefore represent sequential stages in the capacitation process. However, each change takes place at different rates in individual cells, revealing considerable functional heterogeneity within the sperm population. Recent work on membrane changes provoked by cooling has indicated similarities with capacitational changes. The effect of cooling may therefore be to induce premature capacitation (and destabilization). Such an effect would greatly compromise sperm fertilizing potential. A pig sperm-egg interaction model was used to examine quantitative details of zona binding and zona penetrating abilities within capacitated sperm populations, and sperm behaviour was found not to accord with generally held beliefs. In particular, individual spermatozoa that have bound to the zona pellucida show great variation in the delay before penetrating: no evidence has been found for a specially competent subgroup. Even in sperm samples incubated to undergo maximal capacitational membrane changes, cells with actual penetrating potential represent less than $15 \%$ of the total number that attach initially to the zona pellucida. Thus detection of capacitational membrane changes appears greatly to overestimate zona penetrating capability. Future studies linking sperm membrane characteristics with semen fertility in the field will need to consider differences between in vitro and in vivo conditions. The need for survival in the female tract may require much slower sperm responses than are considered optimal for in vitro fertilization.
\end{abstract}

\section{Introduction}

In seeking to preserve semen, the essential goal is obviously to preserve fertilizing ability. However, if definition of the goal is sought in terms of cell biology, i.e. which particular sperm functions need special protection, an answer is much less obvious - indeed, clear answers cannot yet be given. We still lack detailed understanding of the various reproductive processes that affect the fertilizing success or otherwise of individual semen inseminates. Only when we have identified the crucial processes and the way in which they impinge on sperm function are we likely to be able to discover better ways of preserving sperm fertilizing ability.

Inevitably, research on sperm function and semen preservation procedures must rely heavily on in vitro investigations and assessment methods. However, linking any observed biochemical or cellular changes to biological action, even to in vitro fertilization (TVF) success, is very problematical. Linking in vitro findings to the in vivo situation is even more challenging. Many articles have reviewed semen fertility and semen assessment in recent years; of those, Amann (1989), Amann and Hammerstedt (1993), Amann et al. (1993), Saacke et al. (1994), Berger et al. (1996) and Dzuik (1996) are 
especially to be recommended. Rather than reiterate the vast body of relevant work in this area, I have preferred to present in this paper a more personal and selective view of the current position, using results from my laboratory's recent work to illustrate my remarks. First, I shall discuss studies of sperm membrane changes that have been found to occur under IVF conditions. Then I shall present some preliminary quantitative studies of pig sperm-egg interaction in vitro, the findings of which conflict somewhat with current concepts. I shall also discuss some boar sperm characteristics that appear to hinder quantitative comparisons between sperm samples. Finally, I shall propose some overall aspects of sperm behaviour that may play an important role in vivo and that should be considered as we plan our future approaches for assessing sperm fertilizing capability. Limitations of space have resulted in arbitrary choices when referring to others' published findings.

\section{A Brief Overview of Fertilization}

The events and processes involved in the actual act of fertilization have been very well studied in mammals (see Yanagimachi, 1994). The current view is that the fertilizing spermatozoon initiates fertilization by binding in a specific fashion to the egg's zona pellucida envelope. In a process activated by this binding, it exocytoses its acrosome ('acrosome reaction'). Then, with the aid of the action of enzymes exposed as a result of the exocytosis and of the enhanced motive power generated through expression of additional flagellar flexure (hyperactivated motility), the sperm forces its way through the zona pellucida to the perivitelline space beneath. At this point, the sperm attaches to the egg's plasma membrane via specific receptor-ligand interactions in its head equatorial region, the plasma membrane of the sperm begins to fuse with that of the egg, whence the sperm body is adsorbed into the egg and syngamy ensues. From the spermatozoon's point of view, fertilization involves two vital membrane fusion processes - fusion of the sperm head plasma membrane with the underlying outer acrosomal membrane (as the crucial step in the acrosome reaction), and fusion of the sperm plasma membrane with the oolemma during syngamy itself. Biological membranes are intrinsically stable, and require prior architectural modulation and destabilization in order to fuse. In the case of the spermatozoon, the head plasma membrane must remain stable during maturation and throughout subsequent storage in the epididymis. It must also remain stable as the sperm proceeds on its journey up the female tract. Destabilization should only occur in the vicinity of the egg. It is well known that spermatozoa require a priming process known as capacitation in order for them to acquire the ability of immediate interaction with the egg (see Harrison, 1996). While binding to zona components is believed to initiate in the fertilizing spermatozoa the cascade of molecular events that bring about actual membrane fusion during the acrosome reaction (for example Bailey and Storey, 1994; Roldan et al., 1995), current thinking ascribes to capacitation the destabilizing action needed to render the sperm head plasma membrane potentially fusible. Attention is now being focused on capacitation because, clearly, without this priming procedure, the spermatozoa are incapable of responding to any egg stimuli. Moreover, considerable evidence has accrued that capacitation does indeed bring about alterations in the sperm plasma membrane (see Cohen-Dayag and Eisenbach, 1994). It is also worth pointing out that a thorough understanding of capacitation is of practical importance, because capacitation must be achieved during any in vitro testing of sperm fertilizing ability.

\section{Capacitation and Sperm Membrane Changes}

Although the sperm's requirement for capacitation was discovered some 46 years ago, knowledge of the processes involved is still sketchy despite much research effort having been expended on the topic; the many alterations in sperm characteristics that have been described remain essentially phenomenological (see Cohen-Dayag and Eisenbach, 1994). Our lack of success in gaining understanding may in part be ascribed to having taken 'a wrong view' of the process (see Harrison, 1996). 
Insofar as sperm fertility and semen preservation are concerned, there are two important conceptual questions to be posed. First, do spermatozoa respond to capacitating conditions simply as passive targets of an external action exerted on their surfaces, or are positive alterations induced in the innate cellular processes of the spermatozoa? Second, do all spermatozoa undergo capacitation changes in parallel or in synchrony, or are some cells affected more or faster than others? If the latter is the case, obvious supplementary questions concern differences between boars or even ejaculates in their response to capacitating conditions, and to the modulating effects of preservation treatments.

The answers to these questions are now emerging. They are leading to the contention that capacitation is a positive process that involves alterations in dynamic sperm plasma membrane functions. Within the sperm population, individuals respond to capacitating conditions at widely different rates; there appear to be considerable differences between ejaculates, and preservation treatments modulate considerably sperm sample responses.

Some of the evidence for these views comes from recent studies, in which we have been able to profit from the application of flow cytometry to living sperm suspensions to detect changes in individual cells. From such measurements, we have been able to observe trends within the overall population in response to experimental 'capacitating' treatments. Using three different sorts of fluorescent probe, fluo-3 to detect changes in intracellular calcium (Harrison et al., 1993), fluoresceinconjugated lectins to detect changes in surface glycoproteins (Ashworth et al., 1995), and merocyanine to detect changes in plasma membrane lipid architecture (Harrison et al., 1996a), we have found that bicarbonate specifically induces major alterations in these parameters within the sperm population. We have also demonstrated that bicarbonate enhances considerably the development of ability to bind solubilized zona proteins to the sperm head plasma membrane (Harkema et al., 1998). As several studies have demonstrated that bicarbonate is a key effector of capacitation and fertilization in vitro (see Suzuki et al., 1994; Harrison, 1996), the potential relevance of such membrane changes is clear.

Capacitation of freshly ejaculated spermatozoa appears to take a minimum of several hours in most mammalian species studied. While such estimates are based on 'average population response' (i.e. capacitation is detected as having occurred because a sufficient proportion of capacitated spermatozoa have accumulated in the sample to achieve rapid fertilization), the slowness of the overall process implies that it results from a series of sequential events. The bicarbonate-induced changes described above take place on different time scales, hence we believe that they represent different stages in the capacitation sequence. The merocyanine-detectable change (Harrison et al., 1996a) is of particular interest because it takes place within $5 \mathrm{~min}$ of exposing the sperm cells to bicarbonate. We therefore suspect that it represents one of the earliest steps. Merocyanine is a fluorescent lipophilic molecule that is membrane-impermeable but that binds to the outer leaflet of the plasma membrane of intact cells in relation to the degree of lipid disorder in the leaflet. The action of bicarbonate in enhancing the ability of live spermatozoa to bind merocyanine can thus be interpreted as inducing lipid disorder in the external leaflet of the sperm plasma membrane.

Details of the mechanism of this action remain to be elucidated. However, preliminary evidence indicates that the bicarbonate activates adenylyl cyclase (c.f. Okamura et al., 1985; Visconti et al., 1995) to produce increases in intracellular cyclic AMP concentrations (Fig. 1), which in turn stimulate a protein kinase $A$ to phosphorylate an unknown protein component. This protein controls directly or indirectly plasma membrane lipid architecture. The control mechanism appears to be a dynamic one, involving a continual cycle of phosphorylation and dephosphorylation. Protein kinase inhibitors block the increase in merocyanine binding without blocking cAMP increases; a protein phosphatase inhibitor enhances merocyanine binding (Table 1). It is also noteworthy that the bicarbonate induction of merocyanine binding is temperature dependent and reversible, occurring at $38^{\circ} \mathrm{C}$ but not at $25^{\circ} \mathrm{C}$ (see Fig. 1), and declining as the spermatozoa are transferred from $38^{\circ}$ to $25^{\circ} \mathrm{C}$ (Harrison et al., 1996a). Such reversibility is, of course, in accord with the concept of control being mediated through a dynamic phosphorylation-dephosphorylation cycle. Bicarbonate thus exerts a positive effect on intrinsic sperm metabolic processes, whence we deduce that capacitation is a specific, initiated and controllable process rather than a natural continuation of maturation (c.f. 


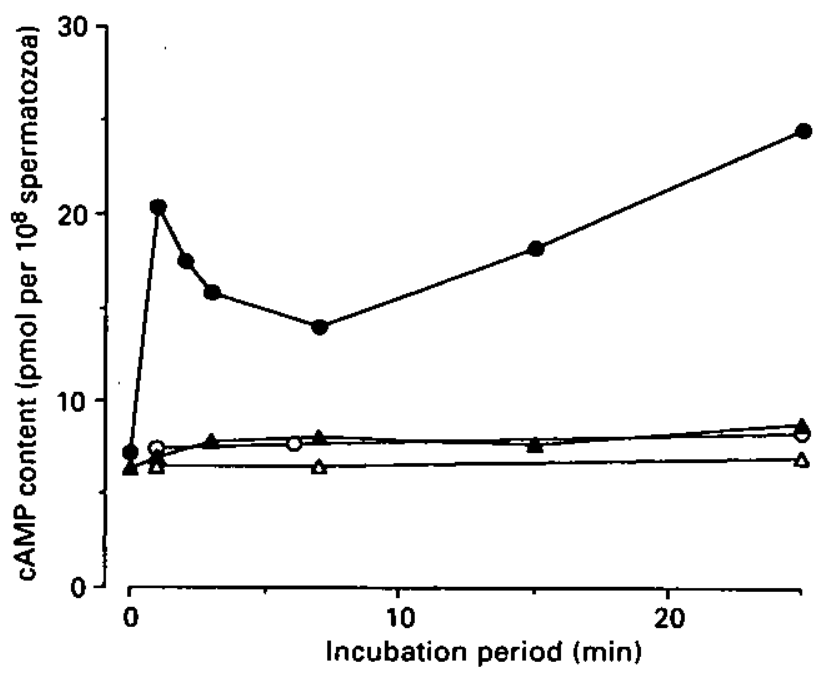

Fig. 1. Cyclic AMP concentrations in boar sperm suspensions exposed to bicarbonate $/ \mathrm{CO}_{2}$. Samples of washed boar spermatozoa were added to variants of a $\mathrm{Ca}^{2+}$-free Hepesbuffered Tyrode's-based medium containing $3 \mathrm{mg} \mathrm{BSA} \mathrm{m}^{-1}$, either at $38^{\circ} \mathrm{C}$ or at $25^{\circ} \mathrm{C}$ (Harrison et al., 1996a). One variant (Tyr +bic) contained 15 mmol bicarbonate $\mathrm{H}^{-1}$ in equilibrium with $5 \% \mathrm{CO}_{2}$; the other (Tyr) contained $15 \mathrm{mmol} \mathrm{NaCl} \mathrm{t}^{-1}$ in place of the bicarbonate and was not exposed to $\mathrm{CO}_{2}$. At intervals after initiating incubation, perchloric acid was added to stop cellular activity ( $0.3 \mathrm{~mol} \mathrm{l}^{-1}$ final concentration), and samples were placed on ice for $30 \mathrm{~min}$. Perchlorate was removed from the protein-free supernatants with tri-noctylamine and freon according to Sharps and McCarl (1982). Cyclic AMP was assayed in the final supernatants using an enzyme-linked immunosorbent assay (Amersham Life Science, Little Chalfont, Bucks). (๑) Tyr +bic at $38^{\circ} \mathrm{C} ;(O)$ Tyr at $38^{\circ} \mathrm{C}$; (A) Tyr +bic at $25^{\circ} \mathrm{C} ;(\Delta)$ Tyr at $25^{\circ} \mathrm{C}$. Note that only Tyr+bic at $38^{\circ} \mathrm{C}$ provokes a rise in cAMP. The effect of these incubation conditions on merocyanine binding (Harrison et al., 1996a) was tested in parallel. Only in $\mathrm{Tyr}+$ bic at $38^{\circ} \mathrm{C}$ were significant numbers of high merocyanine-binding cells induced $(62 \%$ of the total population after $25 \mathrm{~min}$ ); less than $5 \%$ of high merocyanine-binding spermatozoa were found - under other conditions.

These results are from a single experiment; similar cAMP profiles during incubation in Tyr+bic and Tyr at $38^{\circ} \mathrm{C}$ have been found in four other experiments.

Bedford, 1974; Mann and Lutwak-Mann, 1981). It is noteworthy (see Harrison, 1996) that bicarbonate levels are maintained low in the epididymis (where the spermatozoa need to be kept stable), but are high in the female reproductive tract (where capacitation is brought about under natural conditions). Our findings also indicate that capacitation should not be considered as being a release from stabilization conditions within the epididymis (c.f. Bedford, 1991): washed spermatozoa do not readily undergo capacitational changes unless bicarbonate is specifically included in their environment (Harrison et al., 1993; Ashworth et al., 1995; Harrison et al., 1996a).

In linking capacitational changes to fertility, the key point of the bicarbonate-inducible increase in merocyanine binding is that semen samples vary considerably in the quantitative nature of their 
Table 1. Effect of protein kinase and protein phosphatase inhibitors on merocyanine-detectable response to bicarbonate in boar spermatozoa ${ }^{\circ}$

\begin{tabular}{|c|c|c|}
\hline & \multicolumn{2}{|c|}{$\%$ High merocyanine-binding live cells ${ }^{b}$ after } \\
\hline & $10 \mathrm{~min}$ & $25 \mathrm{~min}$ \\
\hline $\begin{array}{l}\text { Expt A: inhibition of protein kinases } \\
5 \text { mmol bicarbonate } \mathrm{l}^{-1} / 1.7 \% \mathrm{CO}_{2} \\
\text { Bicarbonate } / \mathrm{CO}_{2}+1 \mu \mathrm{mol} \text { staurosporine } \mathrm{l}^{-1} \\
\text { Bicarbonate } / \mathrm{CO}_{2}+10 \mu \mathrm{mol} \text { staurosporine } \mathrm{l}^{-1} \\
\text { No addition (control) }\end{array}$ & $\begin{array}{r}43.4 \\
31.6 \\
6.2 \\
1.9\end{array}$ & $\begin{array}{r}39.3 \\
30.4 \\
4.6 \\
3.0\end{array}$ \\
\hline $\begin{array}{l}\text { Expt B: inhibition of protein kinases } \\
6 \mathrm{mmol} \text { bicarbonate } \mathrm{l}^{-1} / 2 \% \mathrm{CO}_{2} \\
\text { Bicarbonate } / \mathrm{CO}_{2}+10 \mu \mathrm{mol} \text { staurosporine } \mathrm{I}^{-1} \\
\text { No addition (control) }\end{array}$ & $\begin{array}{r}59.3 \\
7.3 \\
5.0\end{array}$ & $\begin{array}{r}53.9 \\
9.0 \\
8.4\end{array}$ \\
\hline $\begin{array}{l}\text { Expt } C \text { : inhibition of protein phosphatases } \\
6 \mu \text { mol papaverine } \mathrm{l}^{-1} \\
\text { Papaverine }+1 \mu \mathrm{mol} \text { okadaic acid } \mathrm{l}^{-1} \\
\text { No addition (control) }\end{array}$ & $\begin{array}{r}2.6 \\
20.7 \\
2.9\end{array}$ & $\begin{array}{r}17.3 \\
57.9 \\
6.7\end{array}$ \\
\hline $\begin{array}{l}\text { Expt D: inhibition of phosphatases and kinases } \\
10 \mu \text { mol papaverine } \mathrm{l}^{-1} \\
\text { Papaverine }+1 \mu \text { mol okadaic acid } \mathrm{l}^{-1} \\
\text { Papaverine }+ \text { okadaic acid }+10 \mu \mathrm{mol}^{-1} \text { staurosporine } \mathrm{l}^{-1} \\
\text { No addition (control) }\end{array}$ & $\begin{array}{r}12.5 \\
23.1 \\
6.4 \\
1.6\end{array}$ & $\begin{array}{r}28.5 \\
35.5 \\
5.5 \\
2.5\end{array}$ \\
\hline
\end{tabular}

*Washed boar spermatozoa were added to variants of a Hepes-buffered Tyrode's-based medium at $38^{\circ} \mathrm{C}$; all suspensions contained $3 \mathrm{mg} \mathrm{BSA} \mathrm{ml}^{-1}$, and also Yo-Pro-1 as viability probe. After 10 and 25 min, samples of the suspensions were removed, mixed with merocyanine, and subjected to flow cytometry to detect cellassociated merocyanine and Yo-Pro fluorescence (Harrison et al., 1996a). Because of variation between semen samples in terms of overall response, data from four different experiments are given.

bomputer-generated thresholds, set empirically in each experiment, used the boundary of the major sperm population in the control medium to categorize the limits of low merocyanine fuorescence and low Yo-Pro fluorescence. Cells found beyond these limits were classified as showing high merocyanine-binding or as dead, respectively. Results are expressed as percentages of the total sperm sample.

'Low concentrations of papaverine, a powerful phosphodiesterase inhibitor, were used for sub-maximal stimulation of the CAMP-dependent process (see Harrison et al., 1996a)

response. In some samples, about $80 \%$ of the live cells undergo the change within 10 min of bicarbonate addition; in others; the same treatment results in only a small percentage showing any increase in binding (Harrison et al., 1996b). There is some evidence that the merocyanine-detectable response to bicarbonate may be related to in vivo fertility, although this is still very preliminary. What is intriguing is that storage of spermatozoa in preservation diluents gradually reduces the response, although again individual samples show considerable differences in the degree to which their response may decline (Harrison et al., 1996b).

Another effect of bicarbonate treatment is to increase cell death within the sperm population, apparently by extreme destabilization of the plasma membrane (Harrison et al., 1993). While this destabilization appears to be closely associated with the merocyanine-detectable change (indeed, increased lipid disorder implies increased instability), the cell death observed is not directly related to the degree of merocyanine-detectable response (Fig. 2). When measurements of cell death are combined with measurements of membrane changes in the live sperm population as a response to bicarbonate, a profile with time is obtained that suggests that sperm samples consist of two broad groups, cells that respond rapidly and cells that respond slowly (Fig. 3). It is not yet clear as to which of these two groups may contain the spermatozoa that fertilize in vivo. (This point is made in more detail in a later section.) 


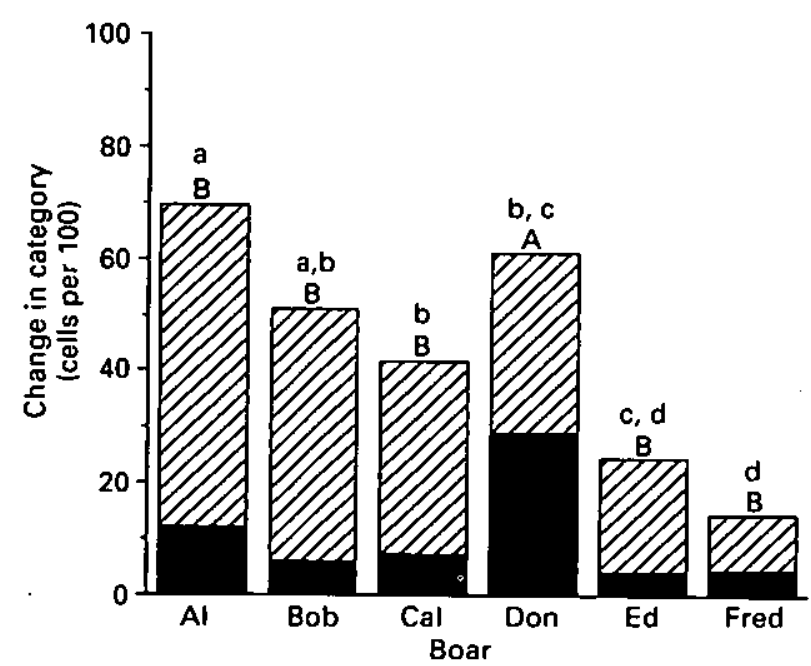

Fig. 2. Variation among boars in sperm response to bicarbonate. Sperm-rich fractions from fresh ejaculates of six different boars were diluted in Beltsville Thawing Solution (Johnson et al., 1988) and kept overnight at ambient temperatures. Spermatozoa were isolated from the extended samples by washing through Percoll and put to incubate at $38^{\circ} \mathrm{C}$ in bicarbonate-containing or bicarbonate-free Hepes-buffered Tyrode's-based medium; YoPro-1 was included as viability probe. After $10 \mathrm{~min}$, samples were removed from the sperm suspensions, mixed with merocyanine, and subjected to flow cytometry. The data were analysed to provide estimates of the relative proportions of high merocyanine-binding live cells and dead cells (see Harrison et al., 1996a for all experimental details). The results are given as the bicarbonate-induced change in either high merocyaninebinding live cells $(C)$ or dead cells $(\square)$, and are the means of four assessments (carried out at weekly intervals); standard error of difference $=6.3$ for changes in merocyanine binding, 5.0 for changes in dead cells. Columns that do not share a common letter are significantly different $(P<0.05)$; lower case letters refer to changes in merocyanine binding, upper case letters to changes in dead cells.

In general terms, however, since capacitation is considered to be a destabilizing process (see Harrison, 1996), any artefactual acceleration of capacitation must be regarded as undesirable. Evidence is now accumulating that treatments associated with cryopreservation may induce capacitation-like changes, at the same time shortening the lifespan of the spermatozoa. The physiological implications of this concept are discussed in more detail in a later section, but of particular interest are the recent demonstrations that cooling can induce changes in the pattern of chlortetracycline (CTC) staining over the sperm head Johnson et al., 1996; Watson, 1996; see also Maxwell and Watson, 1996). These changes closely resemble those seen in capacitating sperm samples, a transition from uniform overall staining of the head (F pattern) to a weak staining of the post-acrosomal region (B pattern), which has been applied to several species as a means of identifying individual capacitated cells (in boar by Wang et al., 1995; Mattioli et al., 1996). Although the precise molecular interpretation of CTC staining has not been elucidated, the fact that both capacitation and cooling induce similar changes in staining pattern strongly suggests that similar destabilizing changes in membrane architecture may have occurred (see Maxwell and Watson, 1996; Watson, 1996). A molecular explanation of the CTC staining changes is clearly urgently needed. 


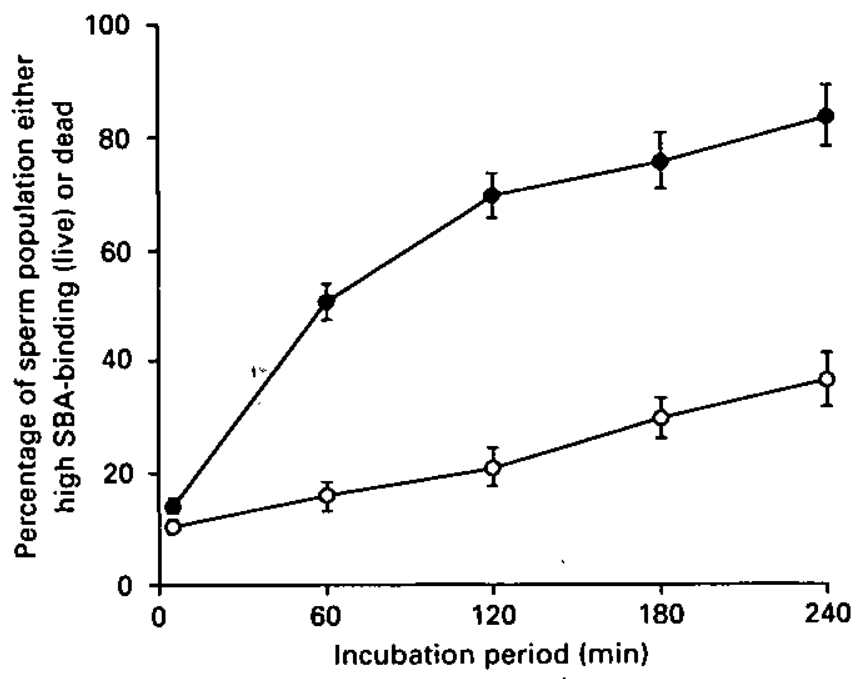

Fig. 3. Effect of bicarbonate on changes in boar sperm samples during lengthy incubation. Washed boar spermatozoa were incubated at $38^{\circ} \mathrm{C}$ in a bicarbonate-containing (O) or a bicarbonatefree $(O)$ Hepes-buffered Tyrode's-based medium with $2 \mathrm{mmol} \mathrm{Ca}^{2+}$ $\mathrm{l}^{-1}$ and $3 \mathrm{mg} \mathrm{BSA} \mathrm{ml}^{-1}$ (Ashworth et al., 1995); propidium iodide was included as viability probe. At intervals, samples were removed from the suspensions, mixed with fluorescein-conjugated soybean agglutinin (F-SBA), and subjected to flow cytometry. The data were analysed to provide estimates of the proportions of high F-SBAbinding live, cells and dead cells (see Ashworth et al., 1995). The results are expressed as the percentage of the total population that were either high F-SBA-binding live or dead (mean \pm SD from nine independent sperm samples). At each time point, the difference between the two media was highly significant $(P<0.001)$.

\section{Membrane Changes and Sperm-Egg Interaction}

Relating specific sperm parameters to fertilizing capability poses great practical problems, particularly in pigs. Laboratory tests compare differences between individual sperm samples, but the fertility of such samples in vivo cannot be assessed in field trials because of the numbers of spermatozoa needed to achieve reasonable fertilization rates (quite apart from such trials being very time-consuming, complex and expensive). However, a satisfactory but simple in vitro test system for assessing sperm-egg interaction remains to be developed. The use of conventional IVF systems (e.g. Matas et al., 1996; Xu et al., 1996) is impractical: lengthy incubation protocols are involved, assessment of categories of spermatozoa other than those that have actually penetrated the egg vitellus is difficult and laborious, and the provision of the matured live eggs needed to obtain the sperm head decondensation within the egg cytoplasm that is the criterion of fertilization is very demanding. There is now a great body of evidence that the zona pellucida provides the major barrier to sperm entry into the egg; the fertilizing ability of a spermatozoon hinges on its ability to cross this barrier (Yanagimachi, 1994; Johnson et al., 1995). Penetration of the zona is thought to require concomitant expression of several sperm functions, including motility (Yanagimachi, 1994; OldsClarke, 1996). Since our ability to determine multiple functions in individual living cells is currently strictly limited, direct measurement of a sperm population's ability to penetrate the zona pellucida would seem to offer at present the best means of assessing fertilizing ability in vitro. Whole eggs are required as test material, since zona penetrating ability cannot realistically be estimated using zona 
Boar ejaculate (usually diluted in BTS)

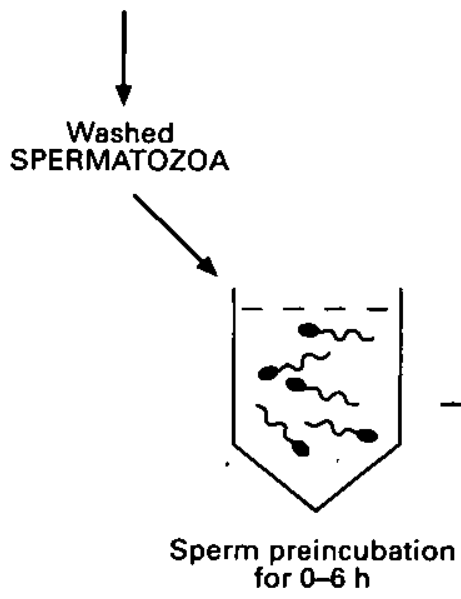

'Capacitation'
Cryopreserved cumulus-free EGGS (immature, from prepubertal gilts)

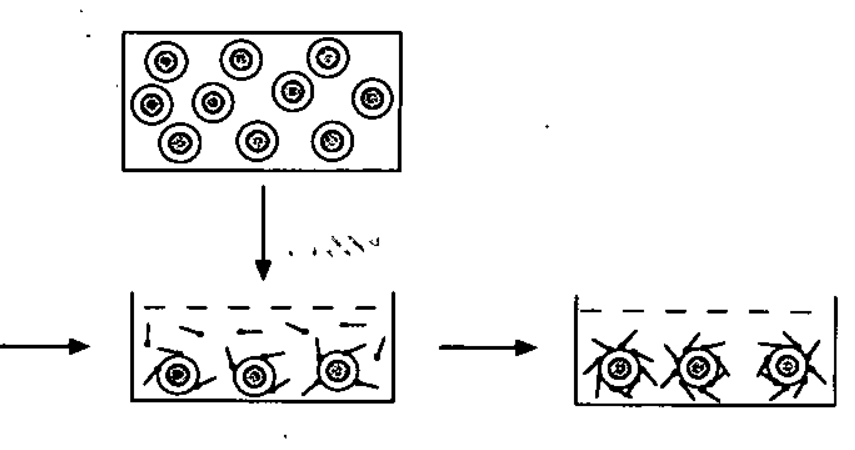

\author{
Further incubation \\ of complex in \\ sperm-free medium \\ for $0-3 \mathrm{~h}$
}
Prolonged binding and
penetration

Fig. 4. Multi-step procedure for testing boar sperm interaction with cryopreserved pig eggs. For details of the procedure, see Lynham and Harrison (1998). Sperm-egg complexes resulting from the possible incubation sequences were subjected to one of three different physical treatments to assess the degree of sperm-egg association. The total number of spermatozoa attached to the egg ('total binding') was assessed by transferring complexes directly into $1 \%(\mathrm{w} / \mathrm{v})$ paraformaldehyde fixative. The number of spermatozoa tightly bound was assessed by removing loosely bound spermatozoa by passaging groups of complexes six times in and out of a specially enlarged 'yellow' Gilson pipette tip before transferring them to fixative. The number of penetrated spermatozoa was assessed by stripping off spermatozoa bound to the zona surface by passaging groups of complexes 12 times in and out of a narrow-bore Gilson Round Gel Loading Tip before transferring them to fixative. After $15 \mathrm{~min}$ in fixative, complexes were washed through a glycine buffer (to quench unreacted formaldehyde) and wet-mounted in a solution containing the nuclear stain Yo-Pro-1. The sperm heads associated with each egg were then counted using fluorescence microscopy.

fragments (i.e. hemi-zonae: Fazeli et al., 1995); for consistency of comparison as well as for convenience, the whole eggs should be available as stored 'pools'.

A number of groups have already used killed eggs successfully to detect zona penetration in several species including pig (Ivanova and Mollova, 1993). On the other hand, doubt has been expressed on the ability of zona penetration tests to inform on fertility in vivo (see Codde and Berger, 1995). However, the systems used so far have not allowed detailed investigation of the different stages of sperm-egg interaction, and the number of eggs have been limited, usually because they have been matured in vitro before storage. In a new approach, therefore, we have used cryopreserved immature eggs isolated in large quantities from the ovaries of prepubertal gilts, to examine separately the stages of sperm function that eventually result in zona penetration (Lynham and Harrison, 1998). In a multi-step model (Fig. 4), the sperm sample is first preincubated in a suitable medium to induce capacitation, then co-incubated briefly with a group of 10-20 eggs to allow a representative subsample of the spermatozoa to bind to the zona surfaces, after which the resultant sperm-egg complexes are transferred to fresh sperm-free medium and incubated further so that the penetrating ability of the already bound spermatozoa can be monitored. Our findings have indicated features of sperm-egg interaction that are not altogether in agreement with current dogma. 

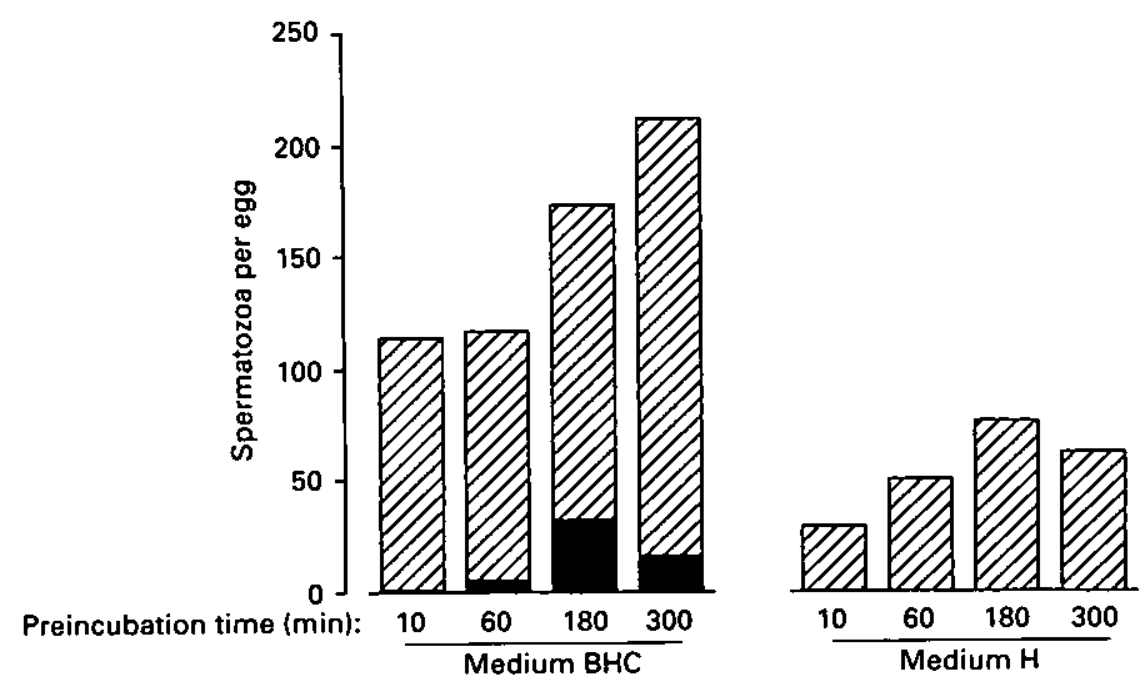

Fig. 5. Development of zona-binding and zona-penetrating abilities during incubation of boar spermatozoa before mixing with eggs. Washed boar spermatozoa $\left(2 \times 10^{6} \mathrm{ml}^{-1}\right)$ were preincubated for various periods, either in medium BHC (known to support fertilization) or in medium $\mathrm{H}$ (known not to support fertilization) (for details of these media, see Suzuki et al., 1994). Samples of the sperm suspensions were then coincubated for $10 \mathrm{~min}$ with groups of cumulus-free pig eggs in the same medium at a concentration of $1.6 \times 10^{6}$ sperm mil. The groups of complexes were then transferred to fresh sperm-free medium, after which some of each group were taken and fixed immediately for assessment of total binding (E), while the others were incubated for a further $60 \mathrm{~min}$ before being repeatedly passaged through a narrow-bore pipette and fixed for assessment of penetration ( $\square$ ). (See Fig. 4 for general explanation of procedures.)

Three replicate experiments were performed with independent semen samples of known high fertility. Each group of eggs yielded a 'mean' data point (spermatozoa per egg) for a particular treatment $x$ replicate. The data were subjected to analysis of variance after $\log$ transformation, and back-transformed means are illustrated. Effects of media and time of preincubation on total binding were both highly significant $(P<0.005)$; effects of media and also of time of preincubation in BHC on penetration were very highly significant $(P<0.001)$.

First, boar spermatozoa bind to the zona pellucida in considerable numbers under noncapacitating conditions. Binding is generally no more than 2-3 times greater under capacitating conditions. While binding ability develops rapidly during preincubation in either capacitating or non-capacitating medium, zona penetrating ability develops slowly and only in capacitating medium (Fig. 5). Thus, although it has been widely monitored in several species as a parameter of fertility (for example in the hemi-zona assay: see Fazeli et al., 1995), zona binding ability does not appear to be a reliable indicator of zona penetrating ability in pigs. Peterson et al. (1984) and Berger et al. (1989) noted the ability of non-capacitated boar spermatozoa to bind to the zona pellucida, but did not at the same time compare zona binding with zona penetrating ability.

A second point of interest is that while we were able to distinguish spermatozoa that were tightly bound to the zona from those that were loosely bound, we were unable to detect any change in the relative proportions of the two types (Fig. 6), either following different periods of preincubation ('capacitation') or during incubation of pre-formed sperm-egg complexes (when numbers of attached spermatozoa remained constant - i.e. attachment was not reversible). Early studies of sperm-zona interaction in hamsters and mice noted transitions in strength of association that could be detected by passaging sperm-egg complexes through wide-bore micropipettes: spermatozoa removed by this 


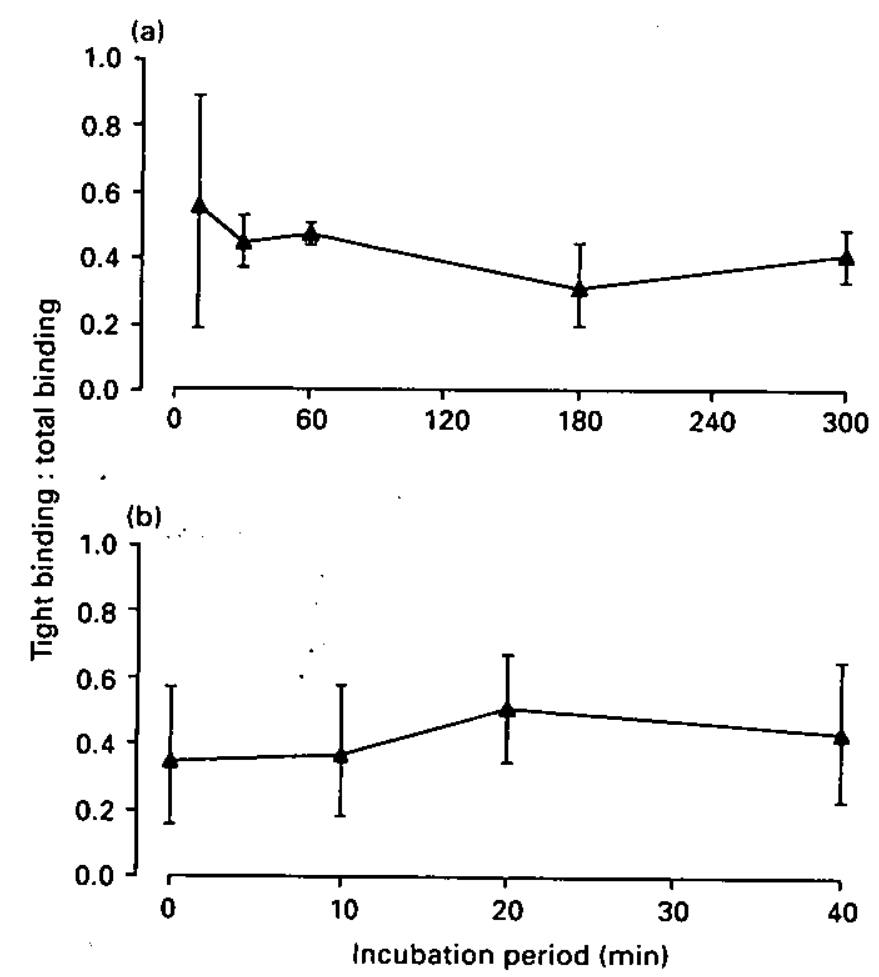

Fig. 6. Strength of boar sperm binding to the zona pellucida during incubation under fertilizing conditions.

(a) Effect of preincubation. Washed spermatozoa were preincubated for various periods in the medium BHC known to support fertilization (see legend to Fig. 5), and then coincubated for $10 \mathrm{~min}$ with groups of eggs in the. same medium. Some of the complexes in each group were then fixed immediately for assessment of total binding while others were passaged through a wide-bore pipette prior to fixation for assessment of tight binding (see legend to Fig. 4).

(b) Effect of incubation of sperm-egg complexes. Washed spermatozoa were preincubated for $3 \mathrm{~h}$ in medium BHC, coincubated with groups of eggs for $10 \mathrm{~min}$, and then the resultant sperm-egg complexes were transferred to fresh sperm-free medium and incubated further. Complexes were removed at intervals after transfer and treated for assessment as before. Each group of eggs yielded a 'mean' data point (spermatozoa per egg). For each time point, the number of spermatozoa tightly bound was divided by the total number of bound sperm to obtain a ratio of tight binding:total binding. Three replicate experiments of each type were performed using independent semen samples of known high fertility. The results were analysed after arcsin transformation, and are presented as back-transformed means \pm SD; differences in binding strength were not significant in either experiment type.

procedure were termed 'attached' while spermatozoa that remained on the zona were termed 'bound' (see Wassarman, 1987). Such transition has been interpreted as the establishment of specific ligand-receptor interactions that lead to the induction of the acrosome reaction (see Wassarman, 1987; Yanagimachi, 1994; Thaler and Cardullo, 1996), and evidence from several species has been presented to indicate that such zona receptor activity increases during capacitation (Leyton and Saling, 1989; Burks et 
al., 1995; Harkema et al., 1998). Our sperm-egg model system does not behave according to this concept. Moreover, when we used fluorescein-conjugated peanut agglutinin to stain (specifically) the acrosomes of zona-bound spermatozoa, we found that sperm-egg complexes from which loosely bound spermatozoa had been removed bore many stained amorphous fragments on their zona surface (Table 2). We concluded that these fragments were acrosomal remnants and deduced that they had been derived from the loosely bound spermatozoa. Since the zona surface is not smooth in relation to a sperm head (e.g. Dietl and Czuppon, 1984; Phillips et al., 1985; Familiari et al., 1992), we believe that spermatozoa often attach by 'embedding' themselves in the rugosities. The strength of attachment of a particular sperm cell more likely reflects the surface area involved in binding rather than the association constant of the particular form of ligand-receptor interaction involved (c.f. Thaler and Cardullo, 1996).

A third finding at odds with dogma concerns the temporal relationship of zona penetration to zona binding. We formed sperm-egg complexes from sperm suspensions that had been preincubated for $3 \mathrm{~h}$ in capacitating medium to develop fully their penetrating ability. After coincubation of spermatozoa and eggs for only $10 \mathrm{~min}$, we transferred the complexes to sperm-free medium and assessed numbers of zona-penetrated spermatozoa after increasing intervals of incubation. Very few penetrated spermatozoa were found in the freshly formed complexes. Numbers of penetrated spermatozoa slowly increased with time in a curvilinear fashion, appearing to reaching a maximum after about $3 \mathrm{~h}$ (Fig. 7). There was no evidence of a rapidly penetrating sperm subgroup. Even after these $3 \mathrm{~h}$ of 'post'incubation, the number of penetrated spermatozoa represented less than $15 \%$ of the total number bound. (Our assay counted all spermatozoa that had entered the zona as 'penetrated'; many fewer would have actually passed through the zona into the perivitelline space.)

These findings need to be viewed against recent studies identifying membrane changes in sperm cells that are specifically engendered by capacitating conditions. The changes, which affect large proportions of the sperm population, have been seen as maximizing in boar sperm samples after some $2 \mathrm{~h}$ of incubation (Ashworth et al., 1995; Harkema et al., 1998). This time course correlates well with other studies based on IVF success which have indicated that optimal capacitation in vitro takes 2-3 $\mathrm{h}$ in pigs (e.g. Yoshida et al., 1993); moreover, in our sperm-egg model also, judged by the number of spermatozoa that were able to penetrate the zona after $1 \mathrm{~h}$ 's postincubation of sperm-egg complexes, zona penetrating ability peaked after $3 \mathrm{~h}$ sperm preincubation. Current thinking (e.g. Wassarman, 1987; Yanagimachi, 1994) considers that the binding of capacitated spermatozoa to the zona pellucida initiates the signalling sequence leading to the acrosome reaction, and that penetration of the zona matrix begins once lytic enzymes have been exposed by the acrosome reaction. In our sperm-egg model, the proportion of zona-bound spermatozoa that actually showed zona penetrating ability was much less than the proportions of spermatozoa reported in previous studies to undergo capacitational changes (e.g. Ashworth et al., 1995; Mattioli et al., 1996; Harkema et al., 1998). Moreover, the time course of the build-up of penetrated spermatozoa indicated varying delays between binding and initiation of penetration, rather than the presence of a subgroup that initiated penetration soon after binding. In other words, the membrane changes induced in spermatozoa by capacitating conditions do not seem to relate directly to the ability to initiate zona penetration on zona contact. Since the latter might arguably be considered as the indicator of true capacitation (see Harrison, 1996), the value of the membrane parameters may need to be reconsidered. Zona penetration appears to demand both the ability to undergo the acrosome reaction in response to zona binding and the expression of vigorous (or even hyperactive) motility (Yanagimachi, 1994; Olds-Clarke, 1996). As the two functions stem from different and essentially independent cellular processes, one might propose that no more than half the cells with one attribute would show the other at the same time; indeed, one could argue that very few at any one time would possess both attributes simultaneously.

\section{Boar Sperm Membrane Properties That May Interfere With Sperm-Egg Interaction In Vitro}

Soon after dispersion in fertilization-supporting medium, boar spermatozoa show a strong tendency to agglutinate head-to-head. This characteristic has been observed previously (Harayama et al., 1994; Suzuki et al., 1994), but its potential effect on the quantitation of sperm-egg interaction in vitro has 


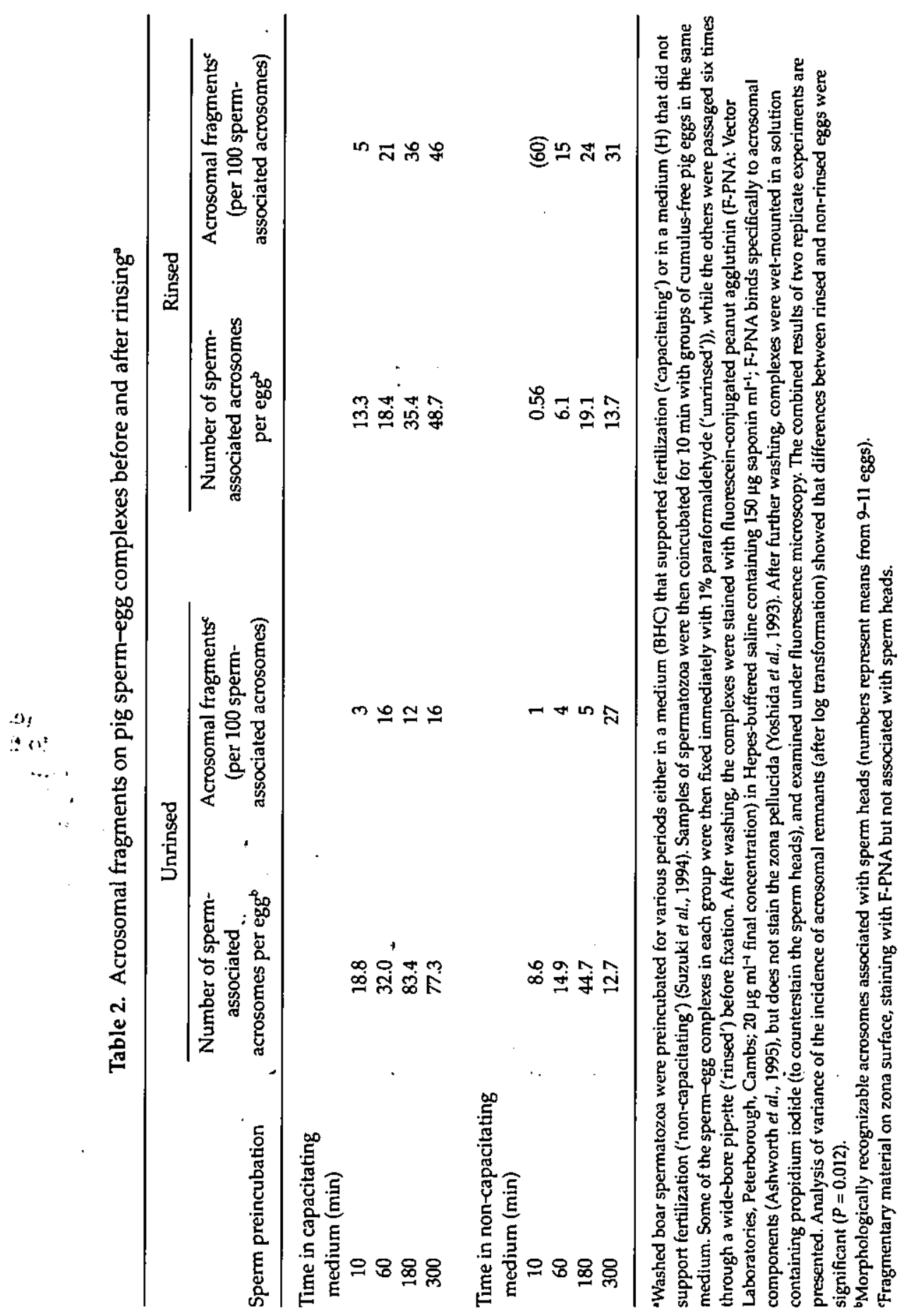




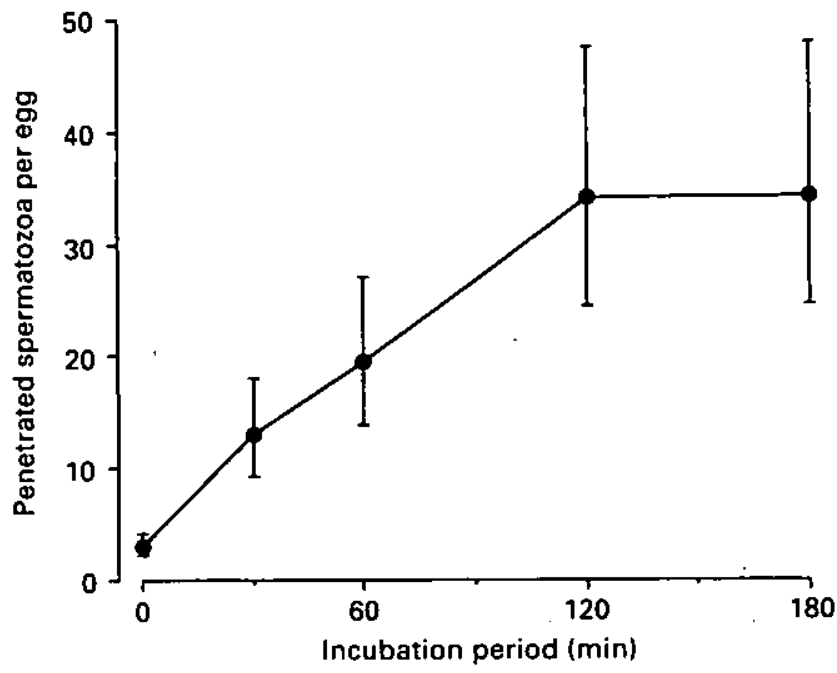

Fig. 7. Zona penetration by boar spermatozoa during incubation of pre-formed sperm-egg complexes. Washed spermatozoa were preincubated for $3 \mathrm{~h}$ in medium $\mathrm{BHC}$, and then coincubated for 10 min with groups of eggs. Sperm-egg complexes were transferred to fresh sperm-free medium, and incubation continued for various periods. Before fixation, some of each group of complexes were passaged through a wide-bore pipette (for assessment of tight binding), while the others were repeatedly passaged through a narrow-bore pipette for assessment of penetration (see legend to Fig. 4). Six replicate experiments were performed using semen samples of known high fertility. Each group of eggs yielded a 'mean' data point (spermatozoa per egg). Because of variation between samples with respect to innate binding capabilities, penetration was calculated as a proportion of the number bound. The data were then subjected to analysis of variance after log transformation. The results are shown as back-transformed means that have been multiplied by the overall mean of numbers bound (to indicate the actual number of spermatozoa involved). Standard deviation limits for these means are also shown. The differences between consecutive time points from 0 to $120 \mathrm{~min}$ were each individually significant $(P<0.05)$.

not been considered. On the one hand, such agglutination causes sedimentation of the spermatozoa, whence concentrations in the vicinity of the eggs (at the bottom of the container) will be enhanced artificially unless the cells are kept in suspension throughout co-incubation. However, agglutination will restrict access of spermatozoa to the eggs, both because agglutinated spermatozoa are effectively sequestered and because the resultant cell groups block mobility of the remaining free cells. The degree of head-to-head agglutination shown by boar spermatozoa under fertilizing conditions can vary greatly from boar to boar.

Another property of boar spermatozoa that could seriously affect quantitative studies of zona penetration is the innate tendency to adhere to the zona (see above). Not only do large numbers of spermatozoa bind under non-capacitating incubation conditions, but the ability to bind varies considerably between samples. Such variation could have disparate effects. On the one hand, more spermatozoa might be expected to penetrate if the number of associated spermatozoa in the sperm-egg complexes were higher. On the other hand, a strong tendency of the overall sperm 
population to bind might impede any enhanced binding by spermatozoa with zona penetrating ability.

Why should boar spermatozoa show a general tendency to bind to the zona pellucida, and why should there be much variation between animals or ejaculates? One possibility is that spermadhesins are responsible (see Berger, 1996). Spermadhesins are members of a family of relatively low molecular weight seminal plasma proteins which coat the spermatozoa and also show strong affinity for the zona pellucida (Töpfer-Petersen and Calvete, 1996). Although some spermadhesins are present in epididymal fluid, most are derived from the seminal vesicles. Spermadhesin coating of spermatozoa increases greatly following ejaculation, when the epididymal cells come into contact with seminal vesicle secretions; it then declines again during capacitation (Dostàlovà et al., 1994). If spermadhesins are indeed responsible for the 'non-specific' zona binding we have observed, they pose a serious problem, because the spermadhesin content of a given sperm sample will depend not only upon the intrinsic spermadhesin levels in that boar but also on the amount of seminal vesicle secretion present in the actual semen sample. This latter will vary, particularly within the sperm-rich fractions that are usually taken for research or for commercial purposes: fractionation of the ejaculate is almost always subjective, and the degree to which the sperm-rich (epididymal) fractions may be mixed with adjoining seminal vesicle contributions is very unpredictable. Differences between boars in terms of ejaculate profile have been presented by Lavon and Boursnell (1975) and Xu et al. (1996); the latter authors also demonstrated differences in fertilizing performance between spermatozoa in the different ejaculate fractions.

Head-to-head agglutination and 'non-specific' zona binding are both manifestations of boar sperm membrane characteristics. They may relate to specific physiological functions within the overall in vivo fertilization process in which an inseminate participates. Immediately, however, either may interfere considerably with quantitative in vitro assessments of sperm ability to interact with the egg. The fact that individual semen samples show great variation in each characteristic is of particular concern. Detailed studies of the two phenomena are required.

\section{Tests of Fertilizing Capability: Future Considerations}

In vitro tests of sperm-egg interaction are needed as a key step in relating specific sperm functional characteristics with fertilizing ability. However, as pointed out in the Introduction, the eventual goal must be to identify those characteristics that are important for fertilizing success in vivo: it is those that must be maintained if semen preservation is to be successful. The conditions under which spermatozoa are induced to fertilize in vitro clearly differ very greatly from the situation in vivo (see discussion in Harrison, 1996). As our knowledge of the overall fertilization process improves, so these differences can be highlighted, to provide a better insight as to what sperm attributes might be advantageous in vivo.

Recent studies have suggested two aspects that merit particular consideration. The first relates to semen longevity in the female reproductive tract. Under natural conditions, sows may be mated at the beginning of oestrus, some $40 \mathrm{~h}$ before ovulation occurs (see Hunter, 1994). Even under conditions of competent husbandry, sows will probably be inseminated 12-24 h before ovulation (see Waberski et al., 1994). The inseminated spermatozoa must therefore remain viable within the female reproductive tract for a considerable period before the eggs become available to them. Investigations in cows, sheep and pigs (see Hunter, 1995) have revealed that, regardless of the time of insemination, the fertilizing spermatozoa do not ascend the upper reaches of the oviduct until shortly before ovulation. Instead, following insemination, the spermatozoa rapidly colonize a specific region of the oviduct, the isthmus, close to the uterotubal junction, from which they are later released to encounter the eggs. The role of the oviductal isthmus as a specific sperm reservoir or storage site is now established for laboratory as well as farm animal species (see Smith and Nothnick, 1997). Hunter (e.g. Hunter, 1995) has proposed that periovulatory signals from the ovary are responsible for modifying the local isthmic environment so as to induce sperm release at this time with concomitant completion of capacitation. Inherent in the concept is protection of 
spermatozoa by the isthmus prior to the engagement of such signals, and indeed oviductal epithelial cells have been shown to exert a strong protective effect on spermatozoa in vitro (Smith and Nothnick, 1997, and references therein). Waberski et al. (1994) have shown that the weil-established decline in fertilization rates following long-term storage of semen under liquid conditions is increasingly pronounced the earlier after onset of oestrus that insemination is performed, but that high fertilization rates continue to be achievable provided insemination is closely synchronized with ovulation. The decline in fertilization rate is accompanied by an even greater decline in the number of accessory spermatozoa found embedded within egg zonae. Linking these observations with the concepts of sperm storage and protection in the oviductal isthmus, it appears that prolonged preservation in a liquid state renders the spermatozoa less able to survive in the female tract. Cryopreservation treatments also appear to compromise sperm survival in the female tract (over and above losses during freezing and thawing). Normal intrauterine insemination of cryopreserved boar semen results in poor fertilization rates, whereas acceptable rates can be achieved by oviductal insemination around the time of ovulation (see Hunter, 1994); similarly, vaginal insemination of cryopreserved ram semen results in very low fertility, whereas uterine insemination achieves satisfactory results (Hunter, 1994; Maxwell and Watson, 1996). A clue to what may be happening during cryopreservation is provided by IVF studies in several species: spermatozoa from frozen semen are able to effect fertilization more rapidly than control spermatozoa from fresh ejaculates, in other words cryopreserved spermatozoa appear prematurely capacitated (see Watson, 1996). Since current beliefs equate capacitation with destabilization (see Harrison, 1996), a shortened lifespan can be expected. At the present time, understanding of the cellular mechanisms that control sperm survival is essentially entirely lacking. There is little doubt that the key organelle is the sperm plasma membrane. Lacking transcriptional or translational activity, the spermatozoon responds to its environment only via the transducing abilities of its plasma membrane, the function of which also controls entirely the internal compartmentation necessary for normal cellular activity. In the long term, therefore, development of improved semen preservation technology will depend on better knowledge of the dynamic functioning of the sperm plasma membrane and the molecular processes that affect its stability. More immediately, however, it is possible that fertility in vivo should not be equated with conventional fertility in vitro. In effect, IVF seeks fertilization as fast as possible (both for practical reasons and because of the detrimental effects of post-ovulatory ageing of the eggs: see Hunter, 1994). However, this will tend to ascribe quantitative 'success' to those sperm samples in which many individual cells undergo capacitation rapidly. As we have seen, such a response may be in direct conflict with the need to survive for long periods in the female tract. It may be, therefore, that our in vitro assessments should include tests of the ability of spermatozoa to survive for long periods under physiological conditions.

At the same time, Hunter's concept of sperm release from the isthmus in response to ovarian signalling (Hunter, 1995) must be coupled with the need for a sufficient supply of capacitated spermatozoa throughout the period of egg release at ovulation. In the case of pigs, the normal complement of 10-20 eggs will be shed over some 2-3 h. Thus, sperm population response to full capacitating conditions should be diverse rather than rapid. In a recent study of the exposure of specific zona-binding sites on the sperm surface under capacitating conditions (Harkema et al., 1998), attention has been drawn to the presence of two subpopulations differing in their speed of response: it was pointed out that the slowly responding subpopulation might represent the spermatozoa that would be more likely to fertilize in vivo. It is noteworthy that in a new study Holt et al. (1997) observed that higher litter size in pigs after artificial insemination may be correlated with lower frequency of both spontaneous and zona pellucida-induced acrosome reactions during in vitro incubation.

\section{Conclusion}

Improvements in semen preservation must focus on preserving the sperm functions that are of paramount importance to the fertilization process. To do so, we need to identify these functions, and 
we need to be able to monitor them by means of simple laboratory tests. The sperm plasma membrane clearly plays a pivotal role in controlling sperm fertilizing ability; not only does it directly mediate the contact interactions between the spermatozoa and the epithelial cells of the female tract and subsequently between the spermatozoa and the egg itself, but it also acts as the receiver of the environmental signals that induce the positive response the spermatozoon must make in order to achieve fertilization. We are still rather far from understanding the relevant aspects of sperm membrane function. However, new knowledge, derived from a wide variety of multidisciplinary approaches, is beginning to reveal key details of the complex processes that enable an inseminate to achieve fertilization. This knowledge should offer the opportunity to derive by logical inference more focused investigations of sperm membrane characteristics as well as more relevant whole-cell approaches to assessing semen fertility. Best progress overall will undoubtedly be achieved by coordinating as closely as possible these two areas of study with further investigations of sperm physiology in the female reproductive tract.

The author would like to record the essential contributions of his colleagues, Nigel Miller, Peter Ashworth, and Jim Lynham with respect to the research results presented in this paper. Semen samples and financial support from the member companies of the UK Association of Pig AI Operators (in conjunction with the BBSRC: Grant 202/A06447), which enabled the studies on stored eggs, are also most gratefully acknowledged.

\section{References}

Amann RP (1989) Can the fertility potential of a seminal sample be predicted accurately? Journal of Andrology $1089-98$

Amann RP and Hammerstedt RH (1993) In vitro evaluation of sperm quality: an opinion Journal of Andrology $14397-406$

Amann RP, Hammerstedt RH and Veeramachaneni DNR (1993) The epididymis and sperm maturation: a perspective Reproduction, Fertility and Development 5 361-381

Ashworth PJC, Harrison RAP, Miller NGA, Plummer JM and Watson PF (1995) Flow cytometric detection of bicarbonateinduced changes in lectin binding in boar and ram sperm populations Molecular Reproduction and Development 40 164-176

Bailey JL and Storey BT (1994) Calcium influx into mouse spermatozoa activated by solubilized mouse zona pellucida, monitored with the calcium fluorescent indicator, fluo-3. Inhibition of the influx by three inhibitors of the zona pellucida induced acrosome reaction: tyrphostin A48, pertussis toxin, and 3-quinuclidinyl benzilate Molecular Reproduction and Development 39 297-308

Bedford JM (1974) Report of a workshop. Maturation of the fertilizing ability of mammalian spermatozoa in the male and female reproductive tract Biology of Reproduction 11 346-362

Bedford JM (1991) The coevolution of mammalian gametes. In A Comparative Overvietv of Mammalian Fertilization pp 3-35 Eds BS Dunbar and MG O'Rand. Plenum Press, New York

Berger T (1996) Fertilization in ungulates Animal Reproduction Science 42 351-360

Berger T, Davis A, Wardrip N and Hedrick JL (1989) Sperm binding to the pig zona pellucida and inhibition by solubilized components of the zona pellucida Journal of Reproduction and Fertility 86 559-586

Berger T, Anderson D and Penedo MCT (1996) Porcine sperm fertilizing potential in relationship to sperm functional capacities Animal Reproduction Science 44 231-239

Burks DJ, Carballada R, Moore HDM and Saling PM (1995) Interaction of a tyrosine kinase from human sperm with the zona pellucida at fertilization Science 269 83-86
Codde JM and Berger T (1995) In vivo fertility of rams in relation to sperm-zona pellucida binding and sperm-zona pellucida penetration of ovine oocytes Theriogenology 44 901-906

Cohen-Dayag A and Eisenbach M (1994) Potential assays for sperm capacitation in mammals American joumal of Physiology $267 \mathrm{C} 1167-\mathrm{C} 1176$

Dietl J and Czuppon AB (1984) Ultrastructural studies of the porcine zona pellucida during the solubilization process by Li-3,5-diiodosalicylate Gamete Research 9 45-54

Dostàlovà Z, Calvete JJ, Sanz I. and Töpfer-Petersen E (1994) Quantitation of boar spermadhesins in accessory sex gland fluids and on the surface of epididymal, ejaculated and capacitated spermatozoa Biochimica et Biophysica Acta $120048-54$

Dzuik PJ (1996) Factors that influence the proportion of offspring sired by a male following heterospermic insemination Animal Reproduction Science 43 63-88

Familiari G, Nottola SA, Macchiarelli G, Micara G, Aragona $C$ and Motta PM (1992) Human zona pellucida during in vitro fertilization: an ultrastructural study using saponin, ruthenium red, and osmium thiocarbohydrazide Molecular Reproduction and Development 32 51-61

Fazeli AR, Holt C, Steenweg W, Bevers MM, Holt WV and Colenbrander B (1995) Development of a sperm hemizona binding assay for boar semen Theriogenology 44 17-27

Harayama H, Miyano T, Miyake M, Kusunoki H and Kato S (1994) Identification of anti-agglutinin for spermatozoa in epididymal boar plasma Molecular Reproduction and Development 37 436-445

Harkema W, Harrison RAP, Miller NGA, Topper EK and Woelders $H$ (1998) Enhanced binding of zona pellucida proteins to the acrosomal region of intact boar spermatozoa in response to fertilizing conditions: a flow cytometric study Biology of Reproduction 58 421-430

Harrison RAP (1996) Capacitation mechanisms, and the role of capacitation as seen in eutherian mammals Reproduction, Fertility and Development 8 581-594

Harrison RAP, Mairet B and Miller NGA (1993) Flow cytometric 
studies of bicarbonate-mediated $\mathrm{Ca}^{2+}$ influx in boar sperm populations Molecular Reproduction and Development 35 197-208

Harrison RAP, Ashworth PJC and Miller NGA (1996a) Bicarbonate $/ \mathrm{CO}_{2}$, an effector of capacitation, induces a rapid and reversible change in the lipid architecture of boar sperm plasma membranes Molecular Reproduction and Development 45 378-391

Harrison RAP, Ashworth PJC and Miller NGA (1996b) Assessment of sperm function under fertilizing conditions Reproduction in Domestic Animals 31 25-30

Holt C, Holt WV, Moore HDM, Reed HCB and Cumock RM (1997) Objectively measured boar sperm motility parameters correlate with the outcomes of on-farm inseminations: results of two fertility trials jourmal of Andrology 18 312-323

Hunter RHF (1994) Causes for failure of fertilization in domestic species. In Embryonic Mortality in Domestic Species pp 1-22 Eds MT Zavy and RD Geisert. CRC Press, Boca Raton, Florida

Hunter RHF (1995) Ovarian endocrine control of sperm progression in the Fallopian tubes Oxford Reviews of Reproductive Biology 17 85-124

lvanova $M$ and Mollova $M$ (1993) Zona-penetration in vitro test for evaluating boar sperm fertility Theriogenology $40397-410$

Johnson LA, Aalbers JG and Grooten HJG (1988) Artificial insemination of swine: fecundity of boar semen stored in Beltsville TS (BTS), Modified Modena (MM), or MR-A and inseminated on one, three and four days after collection Zuchthygiene 23 49-55

Johnson LA, Maxwell WMC, Dobrinsky JR and Welch GR (1996) Staining sperm for viability assessment Reproduction in Domestic Animals 31 37-47

Johnson LR, Pilder SH, Bailey JL and Olds-Clarke P (1995) Sperm from mice carrying one or two $t$ haplotypes are deficient in investment and oocyte penetration Developmental Biology 168 138-149

Lavon U and Boursnell JC (1975) The split cjaculate of the boar: contributions of the epididymides and seminal vesicles Journal of Reproduction and Fertility 42 541-552

Leyton $L$ and Saling P (1989) $95 \mathrm{kd}$ sperm proteins bind ZP3 and serve as tyrosine kinase substrates in response to zona binding Cell 57 1123-1130

Lynham JA and Harrison RAP (1998) The use of stored pig eggs to assess boar sperm fertilizing functions in vitro Biology of Reproduction 58 (in press)

Mann T and Lutwak-Mann C (1981) Male Reproductive Function and Semen pp 26 and 141. Springer-Verlag, Berlin

Matas C, Martinez E, Vazquez JM, Roca J and Gadea J (1996) in vitro penetration assay of boar sperm fertility: effect of various factors on the penetrability of immature pig oocytes Theriogenology 46 503-513

Mattioli M, Barboni B, Lucidi P and Seren E (1996) Identification of capacitation in boar spermatozoa by chlortetracycline staining Theriogenology 45 373-381

Maxwell WMC and Watson PF (1996) Recent progress in the preservation of ram semen Animal Reproduction Science $\mathbf{4 2}$ 55-65

Okamura N, Tajima $Y$, Soejima A, Masuda $H$ and Sugita $Y$ (1985) Sodium bicarbonate in seminal plasma stimulates the motility of mammalian spermatozoa through dinect activation of adenylate cyclase Journal of Biological Chemistry 260 9699-9705

Olds-Clarke P (1996) How does poor motility affect sperm fertilizing ability? Journal of Andrology 17 183-186
Peterson RN, Russell LD and Hunt WP (1984) Evidence for specific binding of uncapacitated boar spermatozoa to porcine zonae pellucidae in vitro. Journal of Experimental Zoology 231 137-147

Phillips DM, Shalgi $R$ and Dekel $N$ (1985) Mammalian fertilization as seen with the scanning electron microscope American joumal of Anatomy 174 357-372

Roldan ERS, Murase T and Shi Q.X (1994) Exocytosis in spermatozoa in response to progesterone and zona pellucida Science 266 1578-1581

Saacke RG, Nadir S and Nebel RL (1994) Relationship of semen quality to sperm transport, fertilization, and embryo quality in ruminants Theriogenology 41 45-50

Sharps ES and McCarl RL (1982) A high performance liquid chromatographic method to measure $5 \mathrm{P}$ incorporation into phosphorylated metabolites in cultured cells Analytical Biochentistry 124 421-424

Smith TT and Nothnick WB (1997) Role of direct contact between spermatozoa and oviductal epithelial cells in maintaining rabbit sperm viability Biology of Reproduction $5683-89$

Suzuki K, Ebihara M, Nagai T, Clarke NGE and Harrison RAP (1994) lmportance of bicarbonate $/ \mathrm{CO}_{2}$ for fertilization of pig oocytes in vitro, and synergism with caffeine Reproduction, Fertility and Development 6 221-227

Thaler CD and Cardullo RA (1996) The initial molecular interaction between mouse sperm and the zona pellucida is a complex binding event Journal of Biological Chemistry 271 23289-23297

Töpfer-Petersen E and Calvete JJ (1996) Sperm-associated protein candidates for primary zona pellucida-binding molecules: structure-function correlations of boar spermadhesins Joumal of Reproduction and Fertility Supplement 50 55-61

Visconti PE, Moore GD, Bailey JL, Leclerc P, Connors SA, Pan D, Oldsclarke P and Kopf GS (1995) Capacitation of mouse spermatozoa. II. Protein tyrosine phosphorylation and capacitation are regulated by a cAMP-dependent pathway Development 121 1139-1150

Waberski D, Weitze KF, Lietmann C, Lübbent zur Lage W, Bortolozzo FP, Willmen T and Petzoldt R (1994) The initial fertilizing capacity of longterm-stored liquid boar semen following pre- and postovulatory insemination Theriogenology 41 1367-1377

Wang WH, Abeydeera LR, Fraser LR and Niwa K (1995) Functional analysis using chlortetracycline fluorescence and in vitro fertilization of frozen-thawed cjaculated boar spermatozoa incubated in a protein-free chemically defined medium Journal of Reproduction and Fertility 104 305-313

Wassarman PM (1987) Early events in mammalian fertilization Annual Review of Cell Biology 3 109-142

Watson PF (1996) Cooling of spermatozoa and fertilizing capacity Reproduction in Donestic Animals 31 135-140

Xu X, Ding J, Seth PC, Harbison DS and Foxcroft GR (1996) In vitro fertilization of in vitro matured pig oocytes: effects of boar and ejaculate fractions Theriogenology 45 745-755

Yanagimachi R (1994) Mammalian fertilization. In The Physiology of Reproduction (2nd Edn) 19 Vol. 1, pp 189-317 Eds E Knobil and JD Neill. Raven Press, New York

Yoshida M, Cran DG and Pursel VG (1993) Confocal and fluorescence microscopic study using lectins of the distribution of cortical granules during the maturation and fertilization of pig oocytes Molecular Reproduction and Development $36462-468$ 